\title{
ECHO SIGNAL SEPARATION USING ICA PROJECTION PURSUIT ALGORITHM
}

\author{
Sanchi Kansra ${ }^{1}$, J Valarmathi' ${ }^{2}$, Vikas Pandey ${ }^{3}$ \\ ${ }^{I}$ School of Electronics and Communication, VIT University, Vellore, India \\ sanchi2791@gmail.com \\ ${ }^{2}$ School of Electronics and Communication, VIT University, Vellore, India \\ jvalarmathi@vit.ac.in \\ ${ }^{3}$ School of Electronics and Communication, VIT University, Vellore, India \\ vickeypandey@gmail.com
}

\begin{abstract}
In any RADAR system, the foremost challenge is to identify the target echo signal from the mixture of unwanted echoes called clutter. RADAR equipment generates linearly frequency modulated pulses which when reflected back gives the information about the target's range, location and velocity of approach. A significant amount of clutter introduced in RADAR signal is generated by the surface of sea. This surface type of clutter is modelled as Weibull noise source which is mixed with target echo. These are treated as two independent sources. Assuming that the echo signals are independent from each other and are non-Gaussian in nature we use Independent Component Analysis's Projection Pursuit Algorithm to separate the components so that these components can be analyzed further for target detection an ranging.
\end{abstract}

Keywords: RADAR, ICA,Blind Source Separation, Weibull Noise.

\section{INTRODUCTION}

The operation of RADAR is simple. It sends a pulse with a particular pulse repetition frequency and observes the echo of that pulse when it bounces off a target. The radar pulse hits several other objects along with target and comes back as different echoes. Clutter is defined as unwanted echo received when radar pulse hits anything apart from target. Clutter is basically of three types:-

1. Point Clutter

2. Surface Clutter

3. Volume Clutter

In any marine navigation system, the major problem is posed by the echo which is reflected over a patch of sea, known as Sea Clutter. The modelling of sea clutter and detection of target in the non -stationary environment of sea is very important.

In this paper we are taking the sea clutter to be modelled as Weibull distribution. To separate the Weibull distributed clutter data from the target echo we are using a blind source separation technique called Independent Component Analysis. The target echo and the Weibull clutter are assumed to be two different sources and they are proved to be independent of each other. Using the projection pursuit ICA algorithm for extracting one source signal we extract the target echo from the mixture of source signals.

\section{SOURCE SIGNALS}

Instead of using a single frequency pulse as radar signal, a linear frequency modulated pulse is used in the radar systems.
Let

$f_{c} \rightarrow$ maximum frequency.

$f_{0} \rightarrow$ starting frequency.

$T_{p} \rightarrow$ Time over which frequency changes.

Then rate of change of the frequency is given by,

$$
k=\frac{f_{c-} f_{0}}{T_{p}}
$$

Hence the generated signal is given by

$$
S_{l f m}(t)=A \cos \left(2 \pi f_{c} t+\pi k t^{2}\right) .
$$

Linear frequency modulated pulse is used to achieve a low range resolution in radar system. For a single frequency pulse range resolution can be defined as:-

$$
R R=\frac{\text { velocity of wave } \times \text { pulse length }}{2}
$$

Where pulse length is the time for which the pulse is being transmitted. For the typical values of pulse length and wave velocity this value is estimated to be between 35-40 meters. Whereas the range resolution for an LFM pulse with wider band of frequencies is defined as:-

$$
R R=\frac{\text { velocity of wave }}{2 \times \text { Bandwidth of LFM signal }}
$$

Using this type of pulse the range resolution can be brought down to as much as 7 meters. 


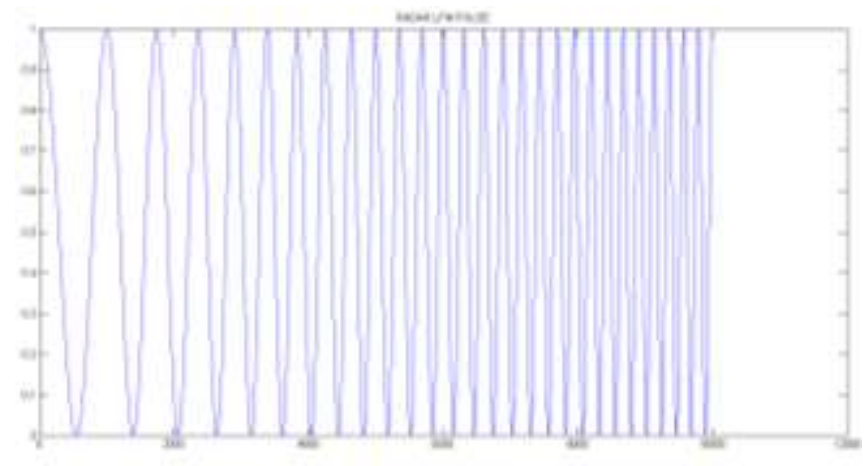

Fig.1. Linear Frequency Modulated Pulse

\section{SEA CLUTTER}

Generally, a mixture of all noises is taken to be a Gaussian noise, as explained by the central limit theorem. The structure to process such data is also easier. But Gaussian estimation works for radar data as long as radar resolution is poor and it has a high grazing angle. For lower grazing angles, the clutter starts becoming spikier and it drifts away from the Gaussian model. For such cases the sea clutter model has to be modeled as a non- Gaussian distribution. Rayleigh distribution or a log normal distribution could be considered but due to the dynamic nature of sea clutter Weibull distribution which is a more generalized form of both Rayleigh and log normal is taken.

The probability density function of Weibull noise can be shown as:-

$$
p(x)=\frac{c}{b}\left(\frac{x}{b}\right)^{c-1} e^{-\left(\frac{x}{b}\right)^{c}}
$$

Where $\mathrm{b}$ is the scale parameter and $\mathrm{c}$ is the shape parameter. Weibull distribution approaches Rayleigh if $\mathrm{c}=2.0$.

\section{INDEPENDENT COMPONENT ANALYSIS}

Let us assume that there is a cocktail party going on in a room. If somebody wants to hear what each guests are talking then he places a microphone in the room and records the conversations. If he listens to the recording he will always hear noise like sounds. Noise like sounds means that they are not noises but combination of voice signals that appear as noise to human ears. This is known as Cocktail Party Effect. Now the question arises as to how to separate those signals. One of the most robust techniques used to perform this operation is 'Independent Component Analysis $(I C A)^{\prime}$.

Assume there are two signals. One of the most important conditions to perform ICA is to assume that the source signals are independent of each other. Let us consider only two signals $S_{1}$ and $S_{2}$. These signals gets mixed linearly as shown:

$\mathrm{X}_{1}=\mathrm{a} \mathrm{S}_{1}+\mathrm{b} \mathrm{S}_{2}$

$\mathrm{X}_{2}=\mathrm{c} \mathrm{S} \mathrm{S}_{1}+\mathrm{b} \mathrm{S}_{2}$
Hence it can be seen that in order to obtain ' $N$ ' number of sources we need ' $N$ ' number of equations. Now practically we receive only signal mixtures. Using just the mixtures we have to obtain the individual components.

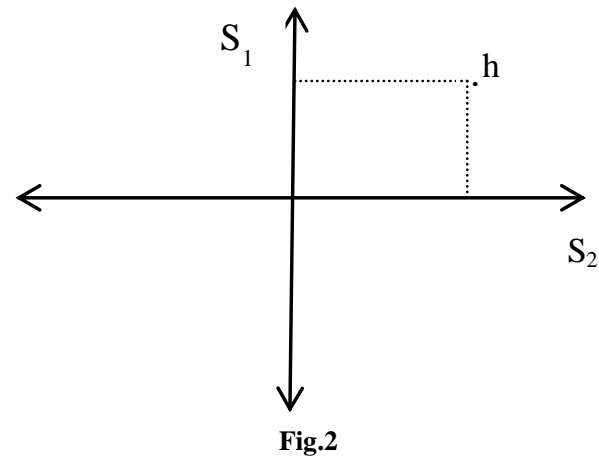

If both the sources are plotted on axis as shown then the point ' $h$ ' shows the amplitude of the both the signals at a time ' $t$ '.

The equations can be written in matrix form as follows.

$\left[\begin{array}{l}\mathrm{x}_{1}(\mathrm{t}) \\ \mathrm{x}_{2}(\mathrm{t})\end{array}\right]=\left[\begin{array}{ll}a & b \\ c & d\end{array}\right]\left[\begin{array}{l}\mathrm{s}_{1} \\ \mathrm{~s}_{2}\end{array}\right]$

$\mathrm{X}=\mathrm{AS}$;

Where, $\mathrm{A}=\left[\begin{array}{ll}a & b \\ c & d\end{array}\right]$ is known as mixing matrix.

In order to find the source components, multiply both the sides by $\mathrm{A}^{-1}$ then we get,

$\left[\begin{array}{l}\mathrm{s}_{1} \\ \mathrm{~s}_{2}\end{array}\right]=\left[\begin{array}{ll}a & b \\ c & d\end{array}\right]^{-1}\left[\begin{array}{l}\mathrm{x}_{1}(\mathrm{t}) \\ \mathrm{x}_{2}(\mathrm{t})\end{array}\right]$

Where, $\mathrm{B}=\left[\begin{array}{ll}a & b \\ c & d\end{array}\right]^{-1}$ is known as Un-mixing matrix. Let the Un-mixing matrix

$\mathrm{B}=\left[\begin{array}{ll}\alpha & \beta \\ \gamma & \delta\end{array}\right]=\left[\begin{array}{ll}a & b \\ c & d\end{array}\right]^{-1}$

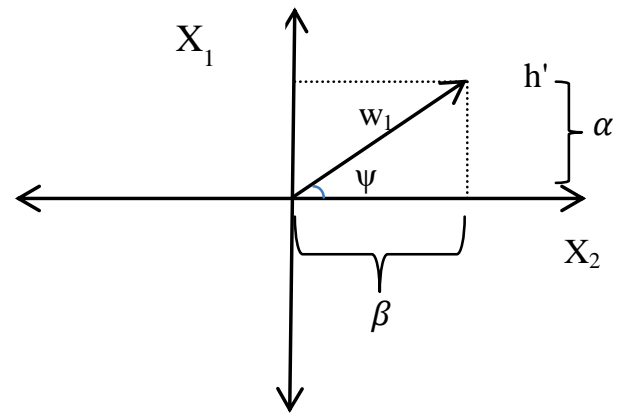

Fig.3

Then to find $S_{1}$ and $S_{2}$ we need to know $\alpha, \beta, \gamma, \delta$ which are unknown as Un-mixing Coefficients. Again similar to the source plot we have to make mixing signals plot as shown below. 
From this point onwards every algorithm that is used in ICA is to find the Un-mixing matrix. The fundamental step in finding mixing matrix is to map the points from S-plane into the X-plane. In figure 2 we see that ' $\mathrm{w}_{1}$ ' is weight vector which defines the source signal $\mathrm{S}_{1}$. Here

$\left|\mathrm{w}_{1}\right|=\sqrt{\alpha^{2}+\beta^{2}}$.

$\Psi=\tan ^{-1}(\alpha / \beta)$.

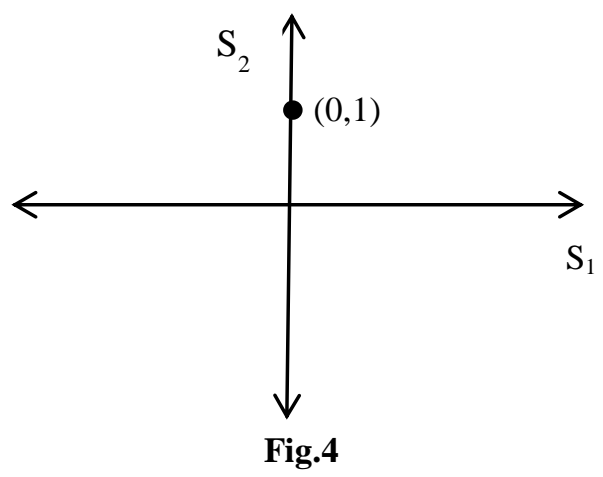

In the above figure 3 , assuming only $\mathrm{S}_{2}$ signal is depicted as $(0,1)$. This can be written as ,

$\mathrm{S}_{2}=\left[\begin{array}{ll}a & b \\ c & d\end{array}\right]\left[\begin{array}{l}0 \\ 1\end{array}\right]=\left[\begin{array}{l}b \\ d\end{array}\right]$

This equation can be understood that $\mathrm{S} 2$ is $(0,1)$ in S-plane then this is mapped as line co-linear with $S_{2}^{\prime}=(b, d)^{\mathrm{T}}$ in $\mathrm{X}$ plane. Similarly $S_{1}^{\prime}=(a, c)^{\mathrm{T}}$. Hence $\mathrm{A}=\left[\begin{array}{ll}S_{1}^{\prime} & S_{2}^{\prime}\end{array}\right]$. It goes without saying that any change in the amplitude of source signal in S-plane gives corresponding changes in X-plane.

Now we will see in general how one source signal gets extracted using ICA.

$\mathrm{S}_{1}=\mathrm{W}_{1}^{\mathrm{T}} \mathrm{X}$

WhereW $_{1}^{\mathrm{T}}$ is a weight vector in $\mathrm{X}$-plane. But

$\mathrm{X}=\mathrm{AS}$

Substituting (9) we get

$\mathrm{S}_{1}=\mathrm{W}_{1}^{\mathrm{T}} \mathrm{AS}$

Or

$\mathrm{S}_{1}=\mathrm{W}_{1}^{\mathrm{T}}\left[\begin{array}{ll}S_{1}^{\prime} & S_{2}^{\prime}\end{array}\right]\left[\begin{array}{ll}S_{1} & S_{2}\end{array}\right]$

One important property to be used here is orthogonality. Inner product of two orthogonal vectors is zero.

If $\mathrm{W}_{1}{ }^{\mathrm{T}}=(\alpha, \beta)$ is orthogonal to $S_{2}^{\prime}=(b, d)^{\mathrm{T}}$ then

$\mathrm{W}_{1}^{\mathrm{T}} S_{2}^{\prime}=0$

But normally it doesn't happen, so

$\mathrm{W}_{1}^{\mathrm{T}} S_{2}^{\prime}=[\alpha, \beta][a, c]^{\mathrm{T}}$ tends to a constant.

$\mathrm{W}_{1}^{\mathrm{T}} S_{1}^{\prime}=k=\left|S_{1}^{\prime}\right|\left|W_{1}\right| \cos \theta$

Where $\theta$ is angle between $S_{1}^{\prime}$ and $W_{1}$. Hence the inference from above derivation is that only when $W_{1}$ is orthogonal to transform axis $S_{1}$, the signal gets extracted otherwise wrong mixture is extracted.

Putting (12),(13) in (11).

$$
\begin{aligned}
& \mathrm{S}_{1}=\left[\begin{array}{ll}
\mathrm{k} & 0
\end{array}\right]\left[\begin{array}{ll}
S_{1} & S_{2}
\end{array}\right]^{\mathrm{T}} \\
= & \mathrm{k} \mathrm{S}_{1}+0 S_{2}
\end{aligned}
$$

$=\mathrm{k} \mathrm{S}_{1}$

which is scaled version of Source Signal.
It can be seen from figure(4) that $\mathrm{W}_{1}$ is orthogonal to $S_{2}^{\prime}$. So the above theory could be summarized as:

- "W" extracts multiple sources, because each row in W is a vector orthogonal to one transformed signal axis $S_{1}^{\prime}$, $S_{2}^{\prime}$.

- Each row in "W" extracts exactly one signal source.

- Rotate each row vector in "W" until the signal extracted by that vector looks like source.

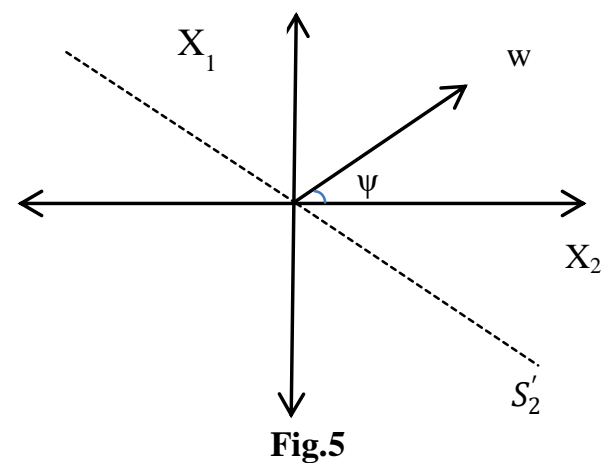

All the above summarized points are keyin implementing ICA.

Suppose that the sources have some properties "P". Assuming that these properties are more in sources than in mixtures, the task is to find set of coefficients that maximize this properties " $\mathrm{P}$ ".

From figure (4), $\mathrm{W}$ is rotated around the origin until these properties are maximized and thus we get our weight vector. But this this is Brute-Force algorithm that requires more number of computations. In case of ICA the properties to be maximized are 'normality', 'independence' and 'complexity'. These properties are defined in terms of "Moments of Probability distribution function (pdf)". Important point to note that the pdf of $\mathrm{X}$ (mixtures) is a Gaussian distribution because of Central Limit Theorem.

\section{PRE PROCESSING}

The kurtosis of a random process is defined as

$$
\mu_{4}=E\left\{\left(x-m_{x}\right)^{4}\right\}
$$

Projection pursuit algorithm requires calculation of the kurtosis in a loop. To simplify the expression we can take the process to be zero mean. In that case the kurtosis can be defined as

$$
\operatorname{kurt}(x)=E\left\{x^{4}\right\}-3\left[E\left\{x^{2}\right\}\right]^{2}
$$

If the process is whitened, i.e.

$$
\begin{gathered}
E\left\{x^{2}\right\}=1 \\
\operatorname{kurt}(x)=E\left\{x^{4}\right\}-3
\end{gathered}
$$

Now, instead of using kurtosis, the fourth moment is directly used, making the process much less hassle.

Whitening means that we linearly transform the observed data vector $\mathrm{x}$

$$
z=V x
$$

Obtained vector $\mathrm{z}$ is white. Next step is how to find out the $\mathrm{V}$ vector which will whiten $\mathrm{x}$. Two very popular techniques used for this purpose are Eigen value decomposition and singular value decomposition. 
Eigen Value Decomposition is applied to covariance matrix of the process. In the case of zero mean process correlation matrix is same as covariance matrix.

$$
E\left\{x x^{T}\right\}=E D E^{T}
$$

Where $\mathrm{E}$ is the Eigen vector matrix of $\mathrm{x}$ and $\mathrm{D}$ is a diagonal matrix containing the Eigen values of $x$.

The whitening matrix $\mathrm{V}$ can be calculated as,

$$
V=E D^{-1 / 2} E^{T}
$$

After whitening the mixing matrix is reformed,

$$
z=V A s=A^{\prime \prime} s
$$

The new whitening matrix is also orthogonal.

$$
E\left\{z z^{T}\right\}=A^{\prime \prime} E\left\{s S^{T}\right\} A^{\prime \prime} T
$$

This means our search for independent components is now restricted to only orthogonal spaces.

\section{PROJECTION PURSUIT ALGORITHM}

One of the robust methods to extract the signals is 'Projection Pursuit Algorithm'. This algorithm seeks one projection at a time. The method of finding the vector is by defining the measure of Non-Gaussanityand then maximizing it. This measure of Non-Gaussanity is known as Kurtosis. So to find a weight vector that maximizes the Kurtosis of extracted signal then,

$\mathrm{Y}=\mathrm{W}^{\mathrm{T}} \mathrm{X}$

Then $\mathrm{Y}$ is the source signal.

When weight vector is rotated around the origin and assuming that each source signal is Super-Gaussian then, $\mathrm{K}$ of extracted $\mathrm{Y}$ is max when $\mathrm{W}$ is orthogonal to projected axes $S_{1}^{\prime}, S_{2}^{\prime}$. This is known as Projection Pursuit Algorithm. This method extracts only one source signal.

In order to extract multiple sources remove the recovered source from the signal mixture and continue the process. This technique is known as Deflation. Gram Schmidt Orthogonalization(GSO) is used to separate the extracted signal from the mixture.

\section{Algorithm:}

1. Number of signals to be extracted

(M).

2. Number of data points $(\mathrm{N})$.

3. $\mathrm{S}_{1}, \mathrm{~S}_{2}, \ldots . . \mathrm{S}_{\mathrm{m}}$ are number of Source Signals.

4. Set Variance of each source to unity.

5. $\mathrm{S}_{\mathrm{m}} \rightarrow \mathrm{S}_{\mathrm{m}}$ '/standard deviation $\left(\mathrm{S}_{\mathrm{m}}\right)$.

6. $\mathrm{S}=\left[\mathrm{S}_{1} ; \mathrm{S}_{2} ; \ldots \mathrm{S}_{\mathrm{m}}\right]$. Forming source matrix.

7. Set a Mixing matrix, $A_{m x m}$

8. Making $\mathrm{m}$ source mixtures, $\mathrm{X}=\mathrm{A} * \mathrm{~S}$. "In practical all the above steps are done by the hardware(eg.Mic).We get only the mixture signals".

9. Sphering/Whitening the mixture signals is to be done using Singular Value Decomposition(SVD).

10. Initialize Un-mixing Vector (w).

11. Normalize the Un-Mixing matrix.

12. Initialize $y=x{ }^{*} z ; ' z$ '-whitened vector.

13. Set number of iterations.

14. Estimate Kurtosis per iteration.

15. Estimate Gradient of Kurtosis for every iteration and the weight vector is updated as follows. $\mathrm{x}_{\text {new }}=\mathrm{x}_{\text {old }}+\eta \mathrm{E}\left[\mathrm{z}\left(\mathrm{x}_{\text {old }} \mathrm{z}^{3} \mathrm{z}\right]\right.$, where $\eta$ is step size for Gradient Ascent.

\section{RESULTS}

The sea clutter data is modeled as Weibull distribution.

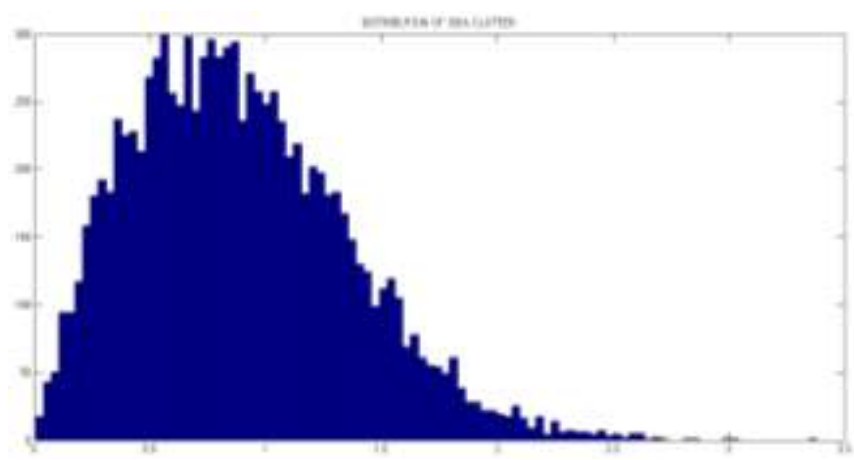

Fig.6

The two sample source signals are first checked for independence.

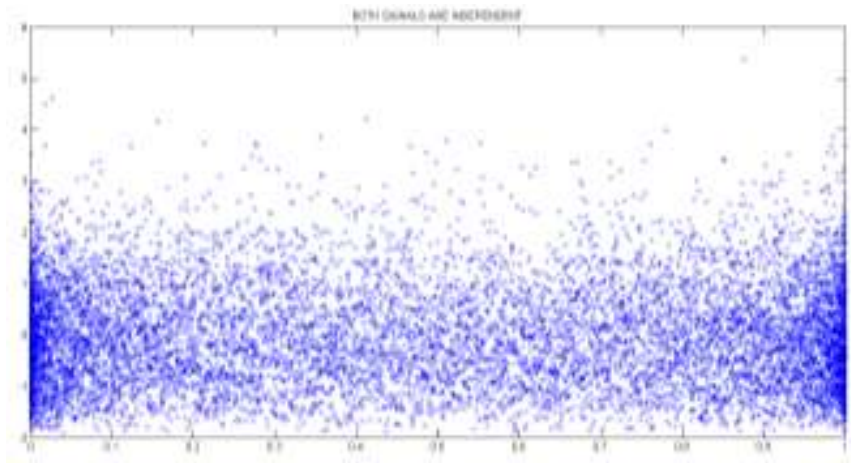

Fig. 7

In the above figure both sources are plotted against each other. Since they have no events which are intersecting so they are independent.

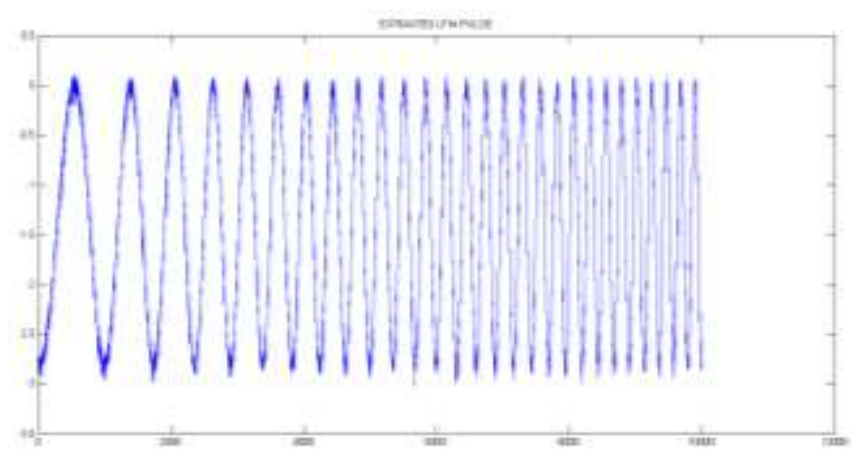

Fig.8

The LFM signal is received here after applying projection pursuit algorithm. Initially the ICA algorithms were majorly used in biomedical applications like in identifying brain waves. In this paper this algorithm has been extended to defense applications. 


\section{CONCLUSION}

The algorithm in this paper proves that when there is mixture of signals received by the receiver. They can be separated using projection pursuit algorithm of Independent Component Analysis. This technique can be used in RADARS for object identification and location. It can also be used in biomedical field to study the brain waves. These techniques can also be extrapolated to study complex mixtures of signals.

\section{REFERENCES}

[1]. "Introduction to RADAR Systems" by Merrill 1 Skolnik .

[2]. "Independent Component Analysis: Algorithm and Applications", by AapoHyvarinen and ErkkiOja.

[3]. "Independent Component Analysis by Minimization of mutual Information" by AapoHyvarinen.

[4]. "Independent Component Analysis" by James V Stone.

[5]. F.A. Fay, J Clarke and R S Peter "Weibull distribution applied to sea clutter" in proceedings of IEEC RADAR “77”,London,UK, 1977.

[6]. "Advanced Sea Clutter Models and their Usefulness for Target Detection" Felix Totir, Emanuel Radoi, Lucian Anton, Cornel Iona, AlexandruSerbanescu, SrdjanStankovic.

[7]. "Adaptive Signal Processing Techniques for Clutter Removal In Radar-based Navigation System" by Yanfeng Liang, Wenwu Wang, Jonathan Chambers, 2009 